\title{
Are linear models really unuseful to describe business cycle data?
}

\section{Artur Silva Lopes and Gabriel Florin Zsurkis}

ISEG, Universidade de Lisboa, Lisboa, Portugal

ABSTRACT

We use first differenced logged quarterly series for the GDP of 29 countries and the euro area to assess the need to use non-linear models to describe business cycle dynamic behaviour. Our approach is model (estimation)-free, based on testing only. We aim to maximize power to detect non-linearities while, simultaneously, avoiding the pitfalls of data mining. The evidence we find does not support some descriptions because the presence of significant non-linearities is observed for two-thirds of the countries only. Linear models cannot be simply dismissed as they are frequently useful. Contrarily to common knowledge, non-linear business cycle variation does not seem to be a universal, undisputable and clearly dominant stylized fact. This finding is particularly surprising for the U.S. case. Some support for non-linear dynamics for some further countries is obtained indirectly, through unit root tests, but this can hardly be invoked to support non-linearity in classical business cycles.
KEYWORDS

Business cycles; non-linear time series models; testing; unit roots

JEL CLASSIFICATION

C22; C51; E32

CONTACT Artur Silva Lopes as|@iseg.utl. ISEG, Universidade de Lisboa, Rua do Quelhas 6, Gab. 107, 1200-781 Lisboa, Portugal ${ }^{1}$ Whom, however, consider that non-linear models are better than linear ones to reproduce some of these features.

${ }^{2}$ Actually, a great effort has been made recently in topics such as unit root testing against non-linear alternatives or in the estimation of new models. Empirically, much effort has been directed recently towards non-linear modelling of interest rates, exchange rates and public finances. 